\title{
Decorous Lower Bounds for Minimum Linear Arrangement
}

\author{
Alberto Caprara \\ Dipartimento di Elettronica, Informatica e Sistemistica, Università di Bologna, I-40136 Bologna, Italy, \\ alberto.caprara@unibo.it \\ Adam N. Letchford \\ Department of Management Science, Lancaster University, Lancaster LA1 4YX, United Kingdom, \\ a.n.letchford@lancaster.ac.uk \\ Juan-José Salazar-González \\ Departamento de Estadística, Investigación Operativa y Computación, Universidad de La Laguna, \\ E-38271 La Laguna, Tenerife, Spain, jjsalaza@ull.es
}

\begin{abstract}
$\mathrm{M}$ inimum linear arrangement is a classical basic combinatorial optimization problem from the 1960s that turns out to be extremely challenging in practice. In particular, for most of its benchmark instances, even the order of magnitude of the optimal solution value is unknown, as testified by the surveys on the problem that contain tables in which the best-known solution value often has one more digit than the best-known lower bound value. In this paper, we propose a linear programming-based approach to compute lower bounds on the optimum. This allows us, for the first time, to show that the best-known solutions are indeed not far from optimal for most of the benchmark instances.

Key words: programming, integer, algorithm, branch and bound; programming, integer, applications; networks; graphs, applications; analysis of algorithms

History: Accepted by Karen Aardal, Area Editor for Design and Analysis of Algorithms; received September 2009; accepted February 2010. Published online in Articles in Advance May 19, 2010.
\end{abstract}

\section{Introduction}

Given a graph $G=(V, E)$, with $V=\{1, \ldots, n\}$, an arrangement (also called a permutation, labelling, ordering, or layout) is a one-to-one function $\psi: V \rightarrow V$. If we view $\psi$ as a placing of the vertices on points $1, \ldots, n$ along the real line, the quantity $|\psi(i)-\psi(j)|$ corresponds to the Euclidean distance between vertices $i$ and $j$. Several important combinatorial optimization problems, collectively known as graph layout problems, call for an arrangement minimizing a function of these distances (see the survey by Díaz et al. 2002). Here, we are concerned with the minimum linear arrangement problem (MinLA for short, using an acronym common in the literature), in which the objective is to minimize the sum of the pairwise distances between all vertices joined by an edge, namely, $\sum_{\{i, j\} \in E}|\psi(i)-\psi(j)|$.

\subsection{Literature Review and State of the Art}

MinLA was originally proposed by Harper (1964). It was proven to be strongly $\mathcal{N} \mathscr{P}$-hard by Garey et al. (1976), and this was later shown to hold even when $G$ is bipartite (Garey and Johnson 1979). For general graphs, the fastest-known exact algorithm (in terms of worst-case performance) is based on dynamic programming and runs in $\mathscr{O}\left(2^{n} \mathrm{~m}\right)$ time (Koren and Harel 2002), where $m:=|E|$. However, MinLA is known to be solvable in polynomial time on trees (Shiloach 1979), outerplanar graphs (Frederickson and Hambrush 1988), and certain Halin graphs (Easton et al. 1996). In fact, for some restricted classes of graphs, optimal layouts are known explicitly (Chung 1988, Harper 1964, Juvan and Mohar 1992, Mitchison and Durbin 1986, Muradyan and Piliposjan 1980).

On the theoretical side, some recent progress has been made on the approximability of MinLA. Approximation algorithms with performance guarantee $\mathscr{O}(\log n)$ were introduced by Bornstein and Vempala (2004) and Rao and Richa (2005). Recently, an $\mathscr{O}(\sqrt{\log n} \log \log n)$ approximation algorithm was found (Charikar et al. 2010, Feige and Lee 2007). It has been shown that there does not exist a polynomialtime approximation scheme for the problem, unless $\mathcal{N} \mathscr{P}$-complete problems can be solved in randomized subexponential time (Ambühl et al. 2007), and it has been conjectured that MinLA cannot be approximated to within a constant factor in polynomial time (Devanur et al. 2006).

On the practical side, the problem appears to be extremely challenging. Before this work (and the 
subsequent work by Caprara et al. 2009, discussed in the conclusions), the best practical method to solve MinLA to proven optimality was the one based on dynamic programming mentioned above (which ruled out the possibility to solve instances with more than, say, 30 vertices). On the one hand, several heuristics were proposed for the problem (Koren and Harel 2002; Petit 2003a, b; Rodriguez-Tello et al. 2008; Safro et al. 2006) and tested on a well-established collection of benchmark instances (Petit 2001). On the other hand, it was so far impossible to certify that their solutions are close to optimal because, with the exception of three instances whose optimum is known by construction, the best lower bounds on the optimum are generally one order of magnitude smaller than the heuristic values.

The situation is illustrated in Petit (2003b), which, among other things, provides a clear picture of the situation concerning the practical solvability of MinLA. Moreover, this is testified in Table 1, in which we report the values of the best-known heuristic solution Best heur, as reported in Rodriguez-Tello et al. (2008), and the best-known lower bound value Best LB, taken from a table in Petit (2003b), along with the associated relative gap. Note that for instances $h c 10$, mesh $33 \times 33$, and bintree 10 , the optimal value is known by the structure of the problem (and reported in column Best heur) and that the lower bound for mesh $33 \times 33$ by the mesh method illustrated in Petit (2003b) is equal to the optimum by definition.

Table 1 Best-Known Solution Values and Lower Bounds for the MinLA Benchmark Instances

\begin{tabular}{lrrrrr}
\hline Name & $n$ & $m$ & Best heur & Best LB & Gap (\%) \\
\hline gd95c & 62 & 144 & 506 & 292 & 42.3 \\
gd96a & 1,096 & 1,676 & 95,242 & 5,155 & 94.6 \\
gd96b & 111 & 193 & 1,416 & 702 & 50.4 \\
gd96c & 65 & 125 & 519 & 241 & 53.6 \\
gd96d & 180 & 228 & 2,391 & 595 & 75.1 \\
c1y & 828 & 1,749 & 62,230 & 14,101 & 77.3 \\
c2y & 980 & 2,102 & 78,757 & 17,842 & 77.3 \\
c3y & 1,327 & 2,844 & 123,145 & 23,417 & 81.0 \\
c4y & 1,366 & 2,915 & 114,936 & 21,140 & 81.6 \\
c5y & 1,202 & 2,557 & 96,850 & 19,217 & 80.2 \\
hc10 & 1,024 & 5,120 & $523,776^{*}$ & 349,525 & 33.3 \\
mesh33 33 & 1,089 & 2,112 & $31,680^{*}$ & 31,680 & 0.0 \\
bintree10 & 1,023 & 1,022 & $3,696^{*}$ & 1,277 & 65.4 \\
randomA1 & 1,000 & 4,974 & 866,968 & 140,634 & 83.8 \\
randomA2 & 1,000 & 24,738 & $6,522,206$ & $4,429,294$ & 32.1 \\
randomA3 & 1,000 & 49,820 & $14,194,583$ & $11,463,259$ & 19.2 \\
randomA4 & 1,000 & 8,177 & $1,717,176$ & 601,130 & 65.0 \\
randomG4 & 1,000 & 8,173 & 140,211 & 39,972 & 71.5 \\
3elt & 4,720 & 13,722 & 357,329 & 44,785 & 87.5 \\
airfoil1 & 4,253 & 12,289 & 272,931 & 40,221 & 85.3 \\
crack & 10,240 & 30,380 & $1,491,126$ & 95,347 & 93.6 \\
whitaker3 & 9,800 & 28,989 & $1,143,645$ & 144,854 & 87.3 \\
\hline
\end{tabular}

Note. Known optimal values are marked by an asterisk.
Table 2 Improved Lower Bounds Found by Our Method for the MinLA Benchmark Instances

\begin{tabular}{lrr}
\hline Name & Our LB & Gap (\%) \\
\hline gd95c & 443 & 12.5 \\
gd96a & 77,860 & 18.3 \\
gd96b & 1,281 & 9.5 \\
gd96c & 402 & 22.5 \\
gd96d & 2,021 & 15.5 \\
c1y & 59,971 & 3.6 \\
c2y & 76,253 & 3.2 \\
c3y & 113,801 & 7.6 \\
c4y & 106,942 & 7.0 \\
c5y & 88,741 & 8.4 \\
bintree10 & 3,696 & 0.0 \\
\hline
\end{tabular}

\subsection{Our Contribution}

In this paper, we focus our attention on the computation of lower bounds for the problem. Our main final contribution is given in Table 2, showing that, for the instances with less than (roughly) 5,000 edges in the benchmark, the current best solutions are very close to fairly close to the optimum, with gaps that are (roughly) between 5\% and $20 \%$. We can also find improved lower bounds for the larger instances, but because our lower bounding procedure does not converge within a reasonable time limit, we preferred not to report the results here.

Our approach is based on the solution of a suitable linear program (LP), which involves variables that represent distances between vertices in the layout and contains exponentially many constraints, handled through separation. In itself, this is a very natural idea that has already been exploited in the literature. However, the key to our approach is, on the one hand, to limit to $m$ the number of variables, and, on the other hand, to work with "strong" constraints that arise from the projection into our variable space of natural inequalities in the space of dimension $\left(\begin{array}{l}n \\ 2\end{array}\right)$ associated with the distances for all vertex pairs. This idea is implicit in Even et al. (2000), in which such an LP was introduced uniquely for theoretical purposes. In this paper, we significantly extend and explore computationally this idea. In doing so, we analyze the structure of the underlying polyhedron, deriving several classes of valid inequalities, proving that they are facet inducing, and discussing the associated separation problems.

The structure of the rest of this paper is as follows. In $\S 2$ we illustrate the LP that we will use, comparing it with the existing LP-based lower bounds for MinLA. In $\S 3$ we associate certain integer polyhedra with MinLA and derive various valid and facetinducing inequalities. The complexity of separation for these inequalities is discussed in $\S 4$. In $\$ 5$ we describe our cutting plane algorithm and the associated computational experiments. Conclusions are given in $\S 6$. 


\subsection{Notation}

Throughout this paper, we use the following customary notation. First of all, as already mentioned, $n$ and $m$ denote the number of vertices and edges, respectively, in $G$. For a given vertex $i, \delta(i)$ denotes the set of edges incident on vertex $i$, and $N(i)$ the set of neighbours of $i$. We also use the standard binomial notation $\left(\begin{array}{l}a \\ b\end{array}\right)=a ! /(b !(a-b) !)$. By subgraph $G^{\prime}$ of $G$ we will always mean an edge-induced subgraph $G^{\prime}=$ $\left(V\left(G^{\prime}\right), E\left(G^{\prime}\right)\right)$ such that $E\left(G^{\prime}\right) \subseteq E$ and $V\left(G^{\prime}\right) \subseteq V$ is the subset of vertices of $G$ that are endpoints of at least one edge in $E\left(G^{\prime}\right)$. For a subgraph $G^{\prime}$ of $G$, we will let LA $\left(G^{\prime}\right)$ denote the optimal MinLA value for $G^{\prime}$. We will sometimes consider the complete graph on $n$ vertices, denoted by $K_{n}=(V, F)$, where $F$ is the set of $\left(\begin{array}{l}n \\ 2\end{array}\right)$ vertex pairs in $V$. In the hope of improving readability, whenever possible $e$ will denote a vertex pair belonging to the edge set $E$ of $G$, whereas $\{i, j\}$ will denote a vertex pair not belonging to $E$. We let $\Psi$ denote the collection of the $n$ ! arrangements of $G$. Finally, given two vertices $i, j \in V$, we will let $\mathscr{P}_{i j}$ denote the collection of the paths (viewed as sets of edges) in $G$ from $i$ to $j$.

\section{Linear Programming Lower Bounds} MinLA is naturally formulated as an integer LP (ILP) as follows. For $i, j \in V$, let the binary variable $x_{i j}$ take the value one if and only if vertex $i$ is placed in position $j$ (i.e., if and only if $\psi(i)=j$ ). Moreover, for $e=\{i, j\} \in E$, let $d_{e} \equiv d_{\{i, j\}}$ be a variable representing the distance between vertices $i$ and $j$, i.e., $|\psi(i)-\psi(j)|$ (whose integrality does not need to be imposed explicitly). A straightforward ILP formulation is

$$
\begin{gathered}
\min \sum_{e \in E} d_{e} \\
\sum_{j \in V} x_{i j}=1, \quad i \in V, \\
\sum_{i \in V} x_{i j}=1, \quad j \in V, \\
d_{\{i, j\}} \geq|p-q|\left(x_{i p}+x_{j q}-1\right), \quad\{i, j\} \in E, p, q \in V, \\
x_{i j} \in\{0,1\}, \quad i, j \in V .
\end{gathered}
$$

A big disadvantage of this formulation, apart from the very large number of constraints, is that its LP relaxation admits the trivial solution $x_{i j}=1 / n$ for all $i, j \in V$, and $d_{i j}=0$ for all $\{i, j\} \in E$, yielding a lower bound of zero. For this reason, the formulation appears to be of no practical use as it stands. However, for the closely related minimum bandwidth problem, suitable variants of this formulation turn out to be the basis of the best practical approaches to tackle the problem (Caprara and Salazar-González 2005). Unfortunately, these methods are heavily based on the fact that the objective function is of bottleneck type and, apparently, cannot be used in the MinLA context (note in particular that these methods carefully avoid solving an LP by a general-purpose solver).

The above formulation is the textbook linearization of the quadratic objective function

$$
\min \sum_{\{i, j\} \in E} \sum_{p, q \in V}|p-q| x_{i p} x_{j q},
$$

subject to the classical assignment constraints on the $x_{i j}$ variables; i.e., MinLA can be seen as a special case of the quadratic assignment problem. Because of the generality and difficulty of the quadratic assignment problem, and the relatively small size of the instances for which the state-of-the-art methods can find a provably optimal solution or even only compute a good lower bound, it appears that tackling MinLA as a quadratic assignment problem is not the best way to proceed (as also suggested by our preliminary computational results, based on the classical linearizationbased lower bounds).

\subsection{A Sparse LP Relaxation}

A natural idea to get lower bounds is to stick to the distance variables $d_{e}$ and to introduce new constraints that these variables must satisfy. In fact, if one does not insist on having a formulation for MinLA, it is natural to get rid of the variables $x_{i j}$, whose only aim is to specify the position of the vertices, significantly reducing the number of variables if the graph is sparse (as is the case for the benchmark instances).

Natural conditions to impose on the distance variables $d_{e}$ are the following rank inequalities. Given a subgraph $G^{\prime}$ of $G$, the rank inequality associated with $G^{\prime}$ imposes that the sum of the distances associated with the edges in $G^{\prime}$ must be at least $\mathrm{LA}\left(G^{\prime}\right)$ (several examples are given in the sequel). Considering a suitable collection $\mathscr{G}$ of subgraphs of $G$, the corresponding LP relaxation is

$$
\begin{aligned}
& \min \sum_{e \in E} d_{e} \\
& \sum_{e \in E\left(G^{\prime}\right)} d_{e} \geq \mathrm{LA}\left(G^{\prime}\right), \quad G^{\prime} \in \mathscr{G} .
\end{aligned}
$$

(Note that the trivial lower bounds $d_{e} \geq 1$ for $e \in E$ are implicit in (1) if all subgraphs induced by a single edge are in 9 .) This LP relaxation was proposed in Liu and Vannelli (1995). Of course, the strength and the solvability of the relaxation depend on $\mathscr{G}$. For instance, if $G \in \mathscr{G}$, the right-hand side of the associated rank inequality is $\operatorname{LA}(G)$, thus solving the relaxation is equivalent to solving MinLA on $G$. On the other hand, if $\mathscr{G}$ is the collection of all subgraphs induced by a single edge, the relaxation has the trivial solution 


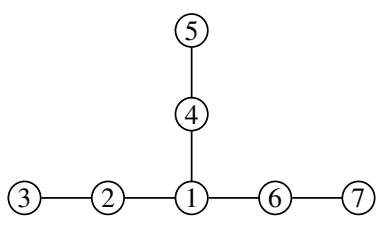

(a)

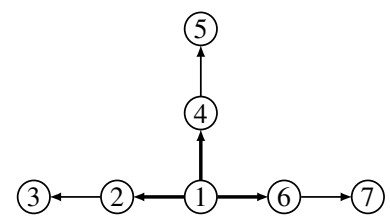

(b)
Figure 1 The Simple Graph $G$ in Example 1 and an Associated Projected Inequality

$d_{e}=1$ for $e \in E$, yielding the useless lower bound $m$ on $\operatorname{LA}(G)$. To get useful lower bounds, it is natural to consider in $\mathscr{G}$ subgraphs $G^{\prime}$ for which not only computation of $\operatorname{LA}\left(G^{\prime}\right)$ is easy but also the separation of the constraints can be handled in practice (possibly via heuristics). A classical and relevant example is the one in which $\mathscr{G}$ contains all the (edge-induced) stars of $G$, noting that for a vertex $i \in V$ there are $2^{|N(i)|}-1$ edge-induced stars, one for each nonempty subset of $N(i)$, all of which may be useful for the LP relaxation. As discussed next, the separation of star inequalities is very easy (and the value $\operatorname{LA}\left(G^{\prime}\right)$ for a star $G^{\prime}$ is given by a simple formula). In Liu and Vannelli (1995), a few other simple structures in addition to stars are considered, even if the separation problem for these is not addressed explicitly, so presumably separation is heuristic in the computational results.

Example 1. Consider the simple graph $G$ with $n=7$ and $m=6$ illustrated in Figure 1(a), for which it is easy to check that $\operatorname{LA}(G)=8$. If we restrict $\mathscr{G}$ to be the collection of the stars of $G$, the nondominated inequalities in (1) are $d_{\{1,2\}}+d_{\{1,4\}}+d_{\{1,6\}} \geq 4$ and the trivial lower bounds $d_{e} \geq 1$ for $e \in E$, and the corresponding optimal value of LP (1) is 7.

\subsection{A Dense LP Relaxation}

The main advantage of LP relaxation (1) is the small number $m$ of variables. However, if one extends the set of distance variables to all $\left(\begin{array}{l}n \\ 2\end{array}\right)$ vertex pairs, one may obtain a much tighter LP relaxation for the same collection of subgraph classes (e.g., if only stars are considered). Recall that $K_{n}=(V, F)$ is the complete graph on $n$ vertices, and let $\mathscr{K}$ now be a suitable collection of edge-induced subgraphs $K^{\prime}$ of $K_{n}$. Let $F\left(K^{\prime}\right)$ denote the set of edges in subgraph $K^{\prime}$. Introduce a binary variable $d_{\{i, j\}}$ for each edge $\{i, j\} \in F$. The LP relaxation, in which only the objective function depends on $G$, is

$$
\begin{gathered}
\min \sum_{e \in E} d_{e} \\
\sum_{\{i, j\} \in F\left(K^{\prime}\right)} d_{\{i, j\}} \geq \operatorname{LA}\left(K^{\prime}\right), \quad K^{\prime} \in \mathscr{K} .
\end{gathered}
$$

For instance, in case $\mathscr{K}$ is the collection of all stars of $K_{n}$, for each vertex $i \in V$, there are $2^{n-1}-1$ inequalities in (2), one for each nonempty subset of $V \backslash\{i\}$.
As it is, LP relaxation (2) has no advantage with respect to (1) because, by setting $d_{\{i, j\}}$ to a sufficiently large value for $\{i, j\} \notin E$, we can satisfy all the constraints when $K^{\prime}$ is not a subgraph of $G$. However, we get an LP relaxation much stronger than (1) if we also impose the natural condition that the $d$ variables define a metric, by the following triangle inequalities:

$$
d_{\{i, j\}} \leq d_{\{i, k\}}+d_{\{k, j\}}, \quad\{i, j, k\} \subseteq V .
$$

An LP relaxation analogous to (2) and (3) was considered in Amaral and Letchford (2006) for a generalization of MinLA.

ExAmple 1 (Continued). If we restrict $\mathscr{K}$ to be the collection of the stars of $K_{n}$, (2) contains, among others, the inequality

$$
d_{\{1,2\}}+d_{\{1,3\}}+d_{\{1,4\}}+d_{\{1,5\}}+d_{\{1,6\}}+d_{\{1,7\}} \geq 12
$$

that, jointly with the triangle inequalities $d_{\{1,3\}} \leq$ $d_{\{1,2\}}+d_{\{2,3\}}, d_{\{1,5\}} \leq d_{\{1,4\}}+d_{\{4,5\}}, d_{\{1,7\}} \leq d_{\{1,6\}}+d_{\{6,7\}}$ and the trivial lower bounds, leads to an optimal value of 7.5 for LP (2) and (3).

For LP relaxation (1), the metric condition can be imposed by the following path inequalities, which are exponentially many but can easily be dealt with as their separation simply requires computing shortest paths in $G$ :

$$
d_{\{i, j\}} \leq \sum_{e \in P_{i j}} d_{e}, \quad\{i, j\} \in E, \quad P_{i j} \in \mathscr{P}_{i j},
$$

recalling that $\mathscr{P}_{i j}$ denotes the collection of all paths in $G$ from $i$ to $j$.

\subsection{A Projected LP Relaxation}

We can combine the advantages of the two previous LP relaxations-namely, the small number of variables of (1) and the relative tightness of (2) and (3) essentially by taking the latter and projecting it onto the variable space of the former. Roughly speaking, this amounts to replacing, in each rank inequality, a variable $d_{\{i, j\}}$ by the sum of the variables $d_{e}$ in the shortest path from $i$ to $j$ in $G$ (and to removing the triangle inequalities). This projected relaxation can be handled in a way analogous to LP (2), in that the separation of rank inequalities is the same modulo precomputing the shortest paths between all vertex pairs, as explained in the following.

Formally, using the same variables as in (1), as well as the same notation as in (2), the projected LP relaxation is

$$
\begin{gathered}
\min \sum_{e \in E} d_{e} \\
\sum_{\{i, j\} \in F\left(K^{\prime}\right)} \sum_{e \in P_{i j}} d_{e} \geq \operatorname{LA}\left(K^{\prime}\right), \\
K^{\prime} \in \mathscr{K},\{i, j\} \in F\left(K^{\prime}\right), P_{i j} \in \mathscr{P}_{i j},
\end{gathered}
$$


where we now have an inequality for each subgraph $K^{\prime} \in \mathscr{K}$ and for each choice of paths in $G$ joining the endpoints of the edges in $K^{\prime}$. Note that these inequalities are not necessarily of rank type, namely, an edge $e$ may appear in more than one path, and therefore $d_{e}$ may have a coefficient larger than one.

Example 1 (Continued). The projected LP relaxation (6) contains, among others, the inequality $2 d_{\{1,2\}}+d_{\{2,3\}}+2 d_{\{1,4\}}+d_{\{4,5\}}+2 d_{\{1,6\}}+d_{\{6,7\}}, \geq 12$, associated with (4) and with the unique paths from one to all vertices, yielding again an optimal value of 7.5 for LP (6). This inequality is illustrated in Figure 1(b), in which the edges with a coefficient of two are drawn as thick lines (the reason for drawing the edges as arrows will be clear in $\S 4$ ).

A formulation equivalent to (6), with $\mathscr{K}$ restricted to be the collection of all stars of $K_{n}$, was given in Even et al. (2000), where the (equivalent) constraints are called spreading constraints and the feasible solutions spreading metrics. In Even et al. (2000), it is observed that, with that choice of $\mathscr{K}$, the LP can be solved in polynomial time. The main result of Even et al. (2000) is that the worst-case ratio between the MinLA optimum and the lower bound given by this LP is $\mathscr{O}(\log n \log \log n)$. In Rao and Richa (2005), it is shown that this ratio is in fact $\Theta(\log n)$.

Proposition 1. The feasible region of LP (6) is the projection over $\mathbb{R}^{E}$ of the feasible region of $L P(2)$ and (3).

Proof. Clearly, for each solution $d^{*}$ of (2) and (3), taking only the components of $d^{*}$ corresponding to the edges in $E$ yields a feasible solution of (6), because the triangle inequalities guarantee that $\sum_{e \in P_{i j}} d_{e}^{*} \geq d_{\{i, j\}}^{*}$ for each vertex pair $\{i, j\} \subseteq V$ and path $P_{i j} \in \mathscr{P}_{i j}$. On the other hand, for each solution $d^{*}$ of (6), it is easy to check that extending this solution to vertex pairs $\{i, j\} \notin V$, by defining $d_{\{i, j\}}^{*}$ to be the length of the shortest path from $i$ to $j$ in $G$ with respect to edge lengths $d^{*}$, yields a feasible solution of (2) and (3).

Corollary 1. LP (6) is equivalent to LP (2) and (3).

The number of constraints of (6) is much larger than the one of (2), as is customary for projections, because exponentially many inequalities in the former correspond to each rank inequality in the latter. However, as we will discuss in detail in $\S 4$, the separation problem for (6) can be polynomially reduced to the separation problem for (2).

\section{A Polyhedral Study}

In this section, we illustrate classes of rank inequalities to be used to concretely define and solve the LP relaxations of $\S 2$. We do this by studying certain integer polyhedra associated with MinLA. We refer the reader to Nemhauser and Wolsey (1988) for an introduction to polyhedral theory and its application to combinatorial optimization.

A polyhedral study of the convex hull of distance vectors representing permutations, i.e., the polyhedron

$P\left(K_{n}\right):=\operatorname{conv}\left\{d \in \mathbb{Z}_{+}^{F}\right.$ : there exists $\psi \in \Psi$ such that

$$
\left.d_{\{i, j\}}=|\psi(i)-\psi(j)| \text { for all }\{i, j\} \in F\right\},
$$

can be found in Amaral and Letchford (2006). (In fact, Amaral and Letchford study a more general class of polyhedra, associated with the so-called single-row facility layout problem, which contains MinLA as a special case.) The affine hull was determined, and several families of valid and facet-inducing inequalities were derived.

Our goal here is to work with only $m$ distance variables $d_{e}$ for each $e \in E$. At first sight, it seems natural to work with the projection of the polytope $P\left(K_{n}\right)$ onto the subspace defined by the edges in $E$, i.e., with the following integer polytope:

$$
\begin{array}{r}
P(G):=\operatorname{conv}\left\{d \in \mathbb{Z}_{+}^{E} \text { : there exists } \psi \in \Psi\right. \text { such that } \\
\left.\qquad d_{\{i, j\}}=|\psi(i)-\psi(j)| \text { for all }\{i, j\} \in E\right\} .
\end{array}
$$

However, $P(G)$ has a fairly complex structure. We have found it helpful to study its dominant, which is the Minkowski sum of $P(G)$ and the nonnegative orthant $\mathbb{R}_{+}^{E}$. That is,

$D(G):=\left\{d \in \mathbb{R}_{+}^{E}\right.$ : there exists $d^{\prime} \in P(G)$ such that $\left.d \geq d^{\prime}\right\}$.

Because the objective function in MinLA is nonnegative, optimizing over $D(G)$ is equivalent to optimizing over $P(G)$. However, $D(G)$ is much easier to work with. Indeed, we have the following three elementary results.

Proposition 2. $D(G)$ is a full-dimensional, unbounded polyhedron.

Proof. Unboundedness is obvious. As to full dimensionality, consider an arbitrary point $d \in D(G)$. By setting, in turn, each component to a sufficiently large value $M$ while leaving the other components unchanged, it is easy to check that the resulting $m$ vectors, belonging to $D(G)$ by definition, together with $d$ form a set of $m+1$ affinely independent vectors.

Recall that an inequality $\alpha^{T} d \geq \beta$ that is valid for $D(G)$ is face inducing if there exists at least one point $d \in D(G)$ such that $\alpha^{T} d=\beta$.

Proposition 3. If the inequality $\sum_{e \in E} \alpha_{e} d_{e} \geq \beta$ is valid for $D(G)$, then $\alpha_{e} \geq 0$ for $e \in E$. Moreover, if it is face inducing, then $\beta \geq \sum_{e \in E} \alpha_{e}$. 
Proof. Nonnegativity of $\alpha$ follows because $D(G)$ is, by definition, of blocking type; i.e., for each $d \in D(G)$, we have $d^{\prime} \in D(G)$ for every $d^{\prime} \geq d$. The lower bound on $\beta$ follows because all components of each $d \in D(G)$ are at least one.

Proposition 4. For each subgraph $G^{\prime}$ of $G$, the inequality $\sum_{e \in E\left(G^{\prime}\right)} \alpha_{e} d_{e} \geq \beta$ is valid (or face inducing or facet inducing) for $D\left(G^{\prime}\right)$ if and only if it is valid (respectively, face inducing or facet inducing) for $D(G)$.

Proof. Note that $D\left(G^{\prime}\right)$ is the projection of $D(G)$ onto the subspace defined by the edges of $G^{\prime}$. The statement is easily checked to be valid in general for a polyhedron of blocking type, its projection onto a subspace, and an inequality having nonzero coefficients only for the variables in this subspace.

When $G=K_{n}$, the relationship between $P(G)$ and $D(G)$ is clear.

Proposition 5. $P\left(K_{n}\right)$ is the unique bounded facet of $D\left(K_{n}\right)$, induced by the equation $\sum_{\{i, j\} \in F} d_{\{i, j\}}=\left(\begin{array}{c}n+1 \\ 3\end{array}\right)$.

Proof. It is shown in Amaral and Letchford (2006) that $P\left(K_{n}\right)$ has dimension $\left(\begin{array}{l}n \\ 2\end{array}\right)-1$ and that its affine hull is defined by the given equation. Because $D\left(K_{n}\right)$ is the dominant of $P\left(K_{n}\right)$ and has dimension $\left(\begin{array}{l}n \\ 2\end{array}\right), P\left(K_{n}\right)$ is a bounded facet of $D\left(K_{n}\right)$. All other facets of $D\left(K_{n}\right)$ must contain half lines and therefore be unbounded.

However, for general graphs $P(G)$ need not even be a face of $D(G)$, as the following example illustrates.

Example 2. Consider the (trivial) graph with $n=3$ and $m=2$, in which $E$ contains the edges $\{1,2\}$ and $\{2,3\}$. The possible values taken by $\left(d_{\{1,2\}}, d_{\{2,3\}}\right)$ in a feasible arrangement are $(1,1),(1,2)$, and $(2,1)$. Thus, $P(G)$ is a triangle in $\mathbb{R}_{+}^{2}$, as shown in Figure 2(a). $D(G)$, on the other hand, has only one extreme point, namely, $(1,1)$, as shown in Figure 2(b).

Going forward, we concentrate on $D(G)$. In the following subsections, we present various valid and facet-inducing inequalities, mainly of rank type, as defined in $\S 2$. Given an inequality that is valid for $D(G)$, we will say that an arrangement $\psi \in \Psi$ is tight for the inequality if the associated distance vector satisfies it at equality. Moreover, we will sometimes use "arrangement" to mean the associated distance vector.

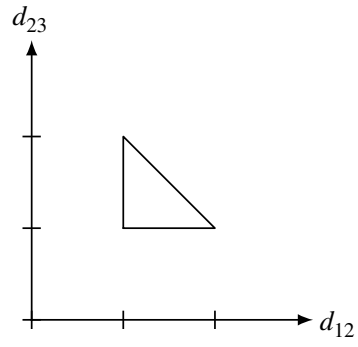

(a)

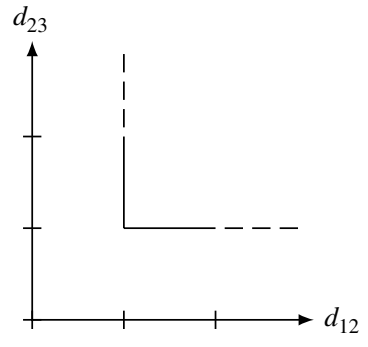

(b)
Figure $2 \quad P(G)$ and $D(G)$ for a Trivial Graph

\subsection{Star Inequalities}

MinLA is trivial when $G$ is a star (i.e., a graph in which all edges are incident on a common vertex). In this case, the optimal MinLA solution has cost $\left\lfloor n^{2} / 4\right\rfloor$. This leads to the following star inequalities.

Proposition 6. For any $i \in V$ and any $S \subseteq N(i)$, the star inequality

$$
\sum_{j \in S} d_{\{i, j\}} \geq\left\lfloor(|S|+1)^{2} / 4\right\rfloor
$$

is valid for $D(G)$ and facet inducing if $|S| \neq 2$.

Proof. By Proposition 4, it suffices to prove the result for the case in which $G$ is itself a star. When $|S|=1$, the star inequality reduces to the trivial lower bound $d_{\{i, j\}} \geq 1$, which is facet inducing because $D(G)=\left\{d_{\{i, j\}} \in \mathbb{R}: d_{\{i, j\}} \geq 1\right\}$. When $|S|=2$, the star inequality is the sum of two trivial lower bounds and therefore not facet inducing.

Now suppose $|S| \geq 3$. Let $\alpha^{T} d=\beta$ be an equation that is satisfied by all arrangements that are tight for the star inequality. Now assume that $|S|$ is even, i.e., $|S|=2 k$ for some integer $k$. Without loss of generality, suppose that $i=k+1$. Now let $\psi$ be the identity arrangement (i.e., $\psi(j)=j$ for all $j$ ) and let $\psi^{\prime}$ be the arrangement obtained by switching vertices 1 and 2, noting that both $\psi$ and $\psi^{\prime}$ are tight. A comparison of the two arrangements shows that $\alpha_{\{1, i\}}=$ $\alpha_{\{2, i\}}$. By symmetry, this shows that $\alpha_{\{i, j\}}$ takes the same value for all $j \in S$. Therefore $\alpha^{T} d \geq \beta$ can be converted into the star inequality by a suitable scaling. The proof for $|S|$ odd is analogous; it suffices to suppose that $i=k$.

Vertex $i$ is called the center of the star. In Amaral and Letchford (2006) it was noted that star inequalities do not in general induce facets of $P\left(K_{n}\right)$.

\subsection{Clique Inequalities}

MinLA is also trivial when $G=K_{n}$, because any arrangement satisfies the equation $d(E)=\left(\begin{array}{c}n+1 \\ 3\end{array}\right)$. This leads to the following clique inequalities.

Proposition 7. For any $n \geq 2$, and for any $S \subseteq V$ inducing a clique in $G$, the clique inequality

$$
\sum_{\{i, j\} \subseteq S} d_{\{i, j\}} \geq\left(\begin{array}{c}
|S|+1 \\
3
\end{array}\right)
$$

is valid and facet inducing for $D(G)$.

Proof. By Proposition 4, we can assume that $G=$ $K_{n}$ and $S=V$. The result then follows from Proposition 5 .

Note that the clique inequalities with $|S|=2$ are the trivial lower bounds $d_{e} \geq 1$. 


\subsection{Circuit Inequalities}

MinLA is also trivial when $G$ is a circuit (i.e., a simple cycle), the optimal MinLA solution having cost $2 n-2$. This leads to the following circuit inequalities.

Proposition 8. For any $C \subseteq E$ inducing a circuit in $G$, the circuit inequality

$$
\sum_{e \in C} d_{e} \geq 2|C|-2
$$

is valid and facet inducing for $D(G)$.

Proof. By Proposition 4, it suffices to prove the result for the case in which $G$ is itself a circuit. Without loss of generality, we assume that $E=$ $\{\{1,2\}, \ldots,\{n-1, n\}\} \cup\{1, n\}$. Let $\alpha^{T} d=\beta$ be an equation that is satisfied by all arrangements that are tight for the circuit inequality. Now let $\psi$ be the identity arrangement, and let $\psi^{\prime}$ be the "shifted" arrangement with $\psi_{i}^{\prime}=i+1 \bmod n$ for $i=1, \ldots, n$, noting that both $\psi$ and $\psi^{\prime}$ are tight. A comparison of the two arrangements shows that $\alpha_{\{1, n\}}=\alpha_{\{n-1, n\}}$. By symmetry, this shows that $\alpha_{\{i, i+1\}}$ takes the same value for all $i$. Therefore $\alpha^{T} d \geq \beta$ can be converted into the circuit inequality by a suitable scaling.

\subsection{Bipartite and Double Star Inequalities}

A class of graphs more general than stars for which there is a closed-form expression for the MinLA optimal value is that of the complete bipartite graphs. We denote the complete bipartite graph by $K_{p, q}$ and assume without loss of generality that $p \leq q$. In Juvan and Mohar (1992) it is shown that the optimal solution to MinLA for $K_{p, q}$ is $r(p, q):=p\left(3 q^{2}+6 p q-p^{2}+4\right) / 12$ (when $p+q$ is even) and $r(p, q):=p\left(3 q^{2}+6 p q-p^{2}+1\right) / 12$ (when $p+q$ is odd). This leads to the following bipartite inequalities.

Proposition 9. Let $S \subseteq V$ and $T \subseteq V \backslash S$ be such that each vertex in $S$ is adjacent to each vertex in $T$, with $p:=$ $|S|, q:=|T|$, and $p \leq q$. The bipartite inequality

$$
\sum_{i \in S} \sum_{k \in T} d_{\{i, k\}} \geq r(p, q)
$$

is valid for $D(G)$. It is facet inducing if and only if one of the following three conditions holds:

- $p=1$ and $q \neq 2$,

- $p=2$ and $q$ is even, or

- $p \geq 3$.

Proof. When $p=1$, the bipartite inequalities reduce to star inequalities and are therefore facet inducing if and only if $q \neq 2$.

Now suppose that $p \geq 3$. By Proposition 4 , it suffices to prove the result for the case in which $G=K_{p, q}$. Let $\alpha^{T} d=\beta$ be an equation that is satisfied by all arrangements that are tight for the bipartite inequality.
In Juvan and Mohar (1992) it was shown that any arrangement of the following form is tight: the first $\lceil(q-p) / 2\rceil$ and the last $\lceil(q-p) / 2\rceil$ positions are occupied by vertices in $T$, whereas the positions in the center are occupied by vertices in $S$ and $T$ in alternation. Notice that if we take such an arrangement and exchange the positions of the vertices in $T$ in positions $\lceil(q-p) / 2\rceil$ and $\lceil(q-p) / 2\rceil+2$, say, $k$ and $l$, we obtain another tight arrangement. Letting $i \in S$ denote the vertex in position $\lceil(q-p) / 2\rceil+1$ and using symmetry, this shows that

$$
\sum_{j \in S \backslash\{i\}} \alpha_{\{j, k\}}=\sum_{j \in S \backslash\{i\}} \alpha_{\{j, l\}}, \quad i \in S, \quad\{k, l\} \subseteq T .
$$

Standard linear algebra calculations show that this is equivalent to

$$
\alpha_{\{i, k\}}=\alpha_{\{i, l\}}, \quad i \in S,\{k, l\} \subseteq T .
$$

Thus, the equation must take the form

$$
\sum_{i \in S} \lambda_{i} \sum_{k \in T} d_{\{i, k\}}=\beta .
$$

Now notice that if we take a tight arrangement of the same form and exchange the positions of the vertices in $S$ in positions $\lceil(q-p) / 2\rceil+1$ and $\lceil(q-p) / 2\rceil+3$, say, $i$ and $j$, we obtain another tight arrangement. The exchange moves vertex $i$ further away from $\lceil(q-p) / 2\rceil$ vertices in $T$ but closer to $q-$ $\lceil(q-p) / 2\rceil-1$ other vertices in $T$. The reverse holds for vertex $j$. This implies that

$$
(p-1) \lambda_{i}=(p-1) \lambda_{j}, \quad\{i, j\} \subseteq S
$$

if $q-p$ is even and

$$
(p-2) \lambda_{i}=(p-2) \lambda_{j}, \quad\{i, j\} \subseteq S
$$

if $q-p$ is odd. These equations imply that $\lambda_{i}=\lambda_{j}$ for all $\{i, j\} \subseteq S$. Thus, the inequality $\alpha^{T} d \geq \beta$ can be converted into the bipartite inequality by a suitable scaling.

A similar argument shows that the bipartite inequality induces a facet when $p=2$ and $q$ is even. When $p=2$ and $q$ is odd, however, the Equations (12) reduce to the trivial equations $0=0$, and we cannot conclude that the bipartite inequality induces a facet. In fact, it does not, because every tight arrangement satisfies the equation

$$
\sum_{k \in T} d_{\{i, k\}}=\sum_{k \in T} d_{\{j, k\}},
$$

where $S=\{i, j\}$.

In the remaining case, in which $p=2$ and $q$ is odd, the bipartite inequality can be derived by summing together two of the following double star inequalities and dividing the resulting inequality by three. 
Proposition 10. Let $\{i, j\} \subseteq V$ and $T \subseteq V \backslash\{i, j\}$ be such that $i$ and $j$ are adjacent to every vertex in $T$, and $|T|$ is odd and at least three. The double star inequality

$$
\sum_{k \in T}\left(2 d_{\{i, k\}}+d_{\{j, k\}}\right) \geq 3\left(|T|^{2}+4|T|-1\right) / 4
$$

is valid and facet inducing for $D(G)$.

Proof. Let $t:=|T|$. By Proposition 4 , it suffices to prove the result for the case in which $G=K_{2, t}$.

Consider any feasible arrangement, and without loss of generality suppose that $i$ is to the left of $j$ (i.e., $\psi(i)<\psi(j))$. Suppose that there are $n_{1}$ vertices to the left of $i$ and $n_{2}$ vertices to the right of $j$ (and therefore $t-n_{1}-n_{2}$ vertices between $i$ and $j$ ). The left-hand side of the double star inequality, computed with respect to the given arrangement, is easily checked to be

$$
2 n_{1}^{2}+n_{2}^{2}-2 n_{1} t-n_{2} t+n_{1}+2 n_{2}+3 t(t+1) / 2 .
$$

A tedious but straightforward calculation shows that this is minimized when $n_{1}=(t-1) / 2$ and $n_{2} \in\{(t-3) / 2,(t-1) / 2\}$, at which points it takes the value $3\left(t^{2}+4 t-1\right) / 4$. Thus, the inequality is valid.

Let $\alpha^{T} d=\beta$ be an equation that is satisfied by all arrangements that are tight for the double star inequality. Exactly the same exchange argument used in the proof of Proposition 9 shows that $\alpha_{\{i, k\}}=\alpha_{\{i, l\}}$ and $\alpha_{\{j, k\}}=\alpha_{\{j, l\}}$ for all $\{k, l\} \subseteq T$. Thus, the equation must take the form

$$
\sum_{k \in T}\left(\lambda d_{\{i, k\}}+\mu d_{\{j, k\}}\right)=\beta
$$

Now let $k$ and $l$ be vertices in T. Consider a tight arrangement in which $k$ lies between $i$ and $j$, and $l$ lies to the immediate right of $j$. If we exchange the positions of $j$ and $l$, we obtain another tight arrangement. A comparison of the two arrangements shows that $\lambda=2 \mu$. Therefore the inequality $\alpha^{T} d \geq \beta$ can be converted into the double star inequality by a suitable scaling.

Double star inequalities are the only inequalities not of rank type addressed in this paper.

\subsection{Tree Inequalities}

All rank inequalities illustrated so far are associated with graphs $G^{\prime}$ for which there is a closed-form expression of $\operatorname{LA}\left(G^{\prime}\right)$. For trees, such an expression is not known but MinLA can be solved efficiently (Shiloach 1979), although the algorithm is far from trivial and the structure of the optimal solution often fairly complex. Therefore, although it appears to be hopeless to prove that they are facet inducing, one may consider the following tree inequalities in a cutting plane approach.
Proposition 11. For any $F \subseteq E$ inducing a tree $T$ in $G$, the tree inequality

$$
\sum_{e \in F} d_{e} \geq \mathrm{LA}(T)
$$

is valid for $D(G)$, and its right-hand side can be computed in polynomial time.

\subsection{Projected Inequalities}

One limitation of the above classes of valid inequalities is that they rely on $G$ containing subgraphs of certain prespecified types (cliques, circuits, and so on). In the following theorem, we describe a "projection" operation that enables one to convert any valid inequality for $D\left(K_{n}\right)$ into one or more valid inequalities for $D(G)$, regardless of the structure of $G$. This is the basic idea behind the definition of LP relaxation (6) from (2).

Proposition 12. Let

$$
\sum_{\{i, j\} \in F} \alpha_{\{i, j\}} d_{\{i, j\}} \geq \beta
$$

with $\alpha$ and $\beta$ nonnegative, be a valid inequality for $D\left(K_{n}\right)$, and $G=(V, E)$ be any connected subgraph of $K_{n}$. Moreover, for each $\{i, j\} \in F$, let $P_{i j} \subseteq E$ be the edge set of an arbitrary path from $i$ to $j$ in $G$. Then the projected inequality

$$
\sum_{\{i, j\} \in F} \alpha_{\{i, j\}} \sum_{e \in P_{i j}} d_{e} \geq \beta
$$

is valid for $D(G)$.

Proof. If the inequality $\sum_{\{i, j\} \in F} \alpha_{\{i, j\}} d_{\{i, j\}} \geq \beta$ is valid for $D\left(K_{n}\right)$, it must also be valid for $P\left(K_{n}\right)$ (because $P\left(K_{n}\right)$ is a facet of $\left.D\left(K_{n}\right)\right)$. Now, for any $\{i, j\} \in F$ and any path $P_{i j}$ from $i$ to $j$, the inequality $\sum_{e \in P_{i j}} d_{e} \geq d_{\{i, j\}}$ is valid for $P\left(K_{n}\right)$, because the distances between points must obey the triangle inequality. This shows that the projected inequality is valid for $P\left(K_{n}\right)$. Because it has nonnegative coefficients, it is also valid for $D\left(K_{n}\right)$. From Proposition 4 , it is also valid for $D(G)$.

The condition that $G$ be connected in Proposition 12 is not a significant limitation, because MinLA decomposes into one or more independent subproblems when $G$ is disconnected. We also remark that there is no guarantee that the projected version of a facetinducing inequality will also be facet inducing, or even only face inducing. This will be discussed in detail for projected star inequalities, anticipating that basically all such inequalities turn out to be face inducing in practice. 


\section{Separation}

Because all of the classes of valid inequalities presented in $\S 3$ are exponential in number, we need exact or heuristic separation algorithms, i.e., routines for detecting when an inequality in a given class is violated by the solution to the current LP relaxation (Nemhauser and Wolsey 1988, Grötschel et al. 1988). In this section, we discuss the complexity of separation for the inequalities introduced in the previous section. Throughout, the LP solution is denoted by $d^{*} \in \mathbb{R}_{+}^{E}$.

\subsection{Separation of Nonprojected Inequalities}

Proposition 13. The separation problem for star inequalities (7) can be solved in $\mathscr{O}(m \log n)$ time.

Proof. It suffices to do the following for each vertex $i \in V$. Sort the vertices $j \in N(i)$ in nondecreasing order of $d_{\{i, j\}}^{*}$. Iteratively insert vertices into $S$ in the sorted order, checking the inequality for violation at each iteration. For a given vertex $i$, the time taken to sort is proportional to $|N(i)| \log |N(i)|$. The total time is therefore proportional to $\sum_{i \in V}|N(i)| \log |N(i)|$, which is $\mathscr{O}(m \log n)$.

Proposition 14. The separation problem for double star inequalities (13) can be solved in $\mathcal{O}(n m \log n)$ time.

Proof. It suffices to do the following for each ordered pair $(i, j) \in V$. Sort the vertices $k \in N(i) \cap N(j)$ in nondecreasing order of $2 d_{\{i, k\}}^{*}+d_{\{j, k\}}^{*}$. Iteratively insert vertices into $T$ in the sorted order, checking the inequality for violation whenever $|T|$ is odd. For a given pair $(i, j)$, the time taken to sort is proportional to $|N(i) \cap N(j)| \log |N(i) \cap N(j)|$, if $N(i) \cap N(j)$ has been precomputed and stored once for all. This implies that the overall time to check, for a given vertex $i$, all pairs $(i, j)$ is $\mathscr{O}(m \log n)$. The total time is then $\mathscr{O}(n m \log n)$.

Proposition 15. The separation problem for clique inequalities (8) is strongly $\mathcal{N} \mathscr{P}$-complete.

Proof. An elementary calculation shows that an inequality (8) violated by $d^{*}$ corresponds to a clique $S^{*}$ in $G$ such that $\sum_{\{i, j\} \subseteq S^{*}}\left(\left|S^{*}\right|+1-3 d_{\{i, j\}}^{*}\right)>0$. We reduce the well-known strongly $\mathcal{N} \mathscr{P}$-complete problem of testing if $G$ has a clique of size $p$ to the problem of finding such a set $S^{*}$ for a suitable $d^{*}$. For $e \in E$, let $d_{e}^{*}:=(p+1-1 / n) / 3$. If $G$ contains a clique $S^{*}$ with $p$ vertices, then we have $\sum_{\{i, j\} \subseteq S^{*}}\left(\left|S^{*}\right|+\right.$ $\left.1-3 d_{\{i, j\}}^{*}\right)=\left(\begin{array}{c}p \\ 2\end{array}\right)(p+1-p-1+1 / n)>0$. On the other hand, for each clique $S^{*}$ with no more than $p-1$ vertices, we have $\sum_{\{i, j\} \subseteq S^{*}}\left(\left|S^{*}\right|+1-3 d_{\{i, j\}}^{*}\right) \leq$ $\left(\begin{array}{c}\left|S^{*}\right| \\ 2\end{array}\right)(p-p-1+1 / n)<0$.

Proposition 16. The separation problem for circuit inequalities (9) is strongly $\mathcal{N} \mathscr{P}$-complete.

Proof. An inequality (9) violated by $d^{*}$ corresponds to a circuit $C^{*}$ in $G$ such that $\sum_{e \in C^{*}}\left(2-d_{e}^{*}\right)>2$. We reduce the well-known strongly $\mathcal{N} \mathscr{P}$-complete problem of testing if $G$ is Hamiltonian to the problem of finding such a $C^{*}$ for a suitable $d^{*}$. For $e \in E$, let $d_{e}^{*}:=2\left(1-1 / n-1 / n^{2}\right)$. If $G$ contains a Hamiltonian circuit $C^{*}$, then we have $\sum_{e \in C^{*}}\left(2-d_{e}^{*}\right)=2 n(1 / n+$ $\left.1 / n^{2}\right)>2$. On the other hand, for all circuits $C^{*}$ with no more than $n-1$ edges, we have $\sum_{e \in C^{*}}\left(2-d_{e}^{*}\right) \leq$ $2(n-1)\left(1 / n+1 / n^{2}\right)<2$.

For bipartite inequalities, we are also convinced that separation is difficult, although we did not find a formal proof.

CONJecture 1. The separation problem for bipartite inequalities (10) is strongly $\mathcal{N} \mathscr{P}$-complete.

For the complexity of separation of tree inequalities we do not dare to conjecture, given the very complex form of the right-hand side.

Heuristic algorithms for the separation of inequalities (8), (9), (10), (14) are illustrated in §5.1.

\subsection{Separation of Projected Inequalities}

As anticipated in $\$ 2$, in this section we discuss the separation of the projected version of the various inequalities that we consider.

Proposition 17. Any polynomial-time algorithm for the separation of a class of valid inequalities (15) for $D\left(K_{n}\right)$ yields a polynomial-time algorithm for the separation of the associated projected inequalities (16).

Proof. Given $d^{*} \in \mathbb{R}^{E}$, suppose that there is an inequality (16) violated by $d^{*}$. Letting $P_{i j}^{*}$ denote the shortest path in $G$ between $i$ and $j$ (with respect to lengths $\left.d_{e}^{*}\right)$, the inequality (16) associated with these shortest paths is also violated. Accordingly, we can proceed as follows. We compute the $P_{i j}^{*}$ paths by an all-pair shortest-path algorithm, let $d_{\{i, j\}}^{* *}$ be the length of $P_{i j}^{*}$ for all vertex pairs $\{i, j\} \in F$, and run the separation algorithm for (15) on this new $d^{* *} \in \mathbb{R}^{F}$ (note in particular that we may have $d_{e}^{* *}<d_{e}^{*}$ for some $e \in E$, meaning that the corresponding path inequality (5) is violated). If a violated inequality is found, the associated violated inequality (16) is easily obtained by replacing each edge $\{i, j\} \in F$ by the edges in $P_{i j}^{*}$.

In other words, given $d^{*} \in \mathbb{R}^{E}$, the separation of the projected inequalities is obtained by defining the complete graph $K_{n}$, with edge lengths $d^{* *}$ equal to the shortest paths in $G$ with respect to $d^{*}$, and then running the separation algorithm over this complete graph. Given that $d^{* *}$ can be found once for all inequalities, the time complexities in the following corollaries do not take into account the associated shortest-path computation.

COROLlaRy 1. The separation problem for projected star inequalities can be solved in $\mathscr{O}\left(n^{2} \log n\right)$ time. 
Corollary 2. The separation problem for projected double star inequalities can be solved in $\mathcal{O}\left(n^{3} \log n\right)$ time.

Note that the converse of Proposition 17 does not hold in general; i.e., the separation of the projected version of a class of inequalities may be easier than the separation of the nonprojected version, given that in the former case the edge lengths $d^{* *}$ satisfy the triangle inequalities. This is not the case, however, for the circuit inequalities.

COROLlaRy 3. The separation problem for projected circuit inequalities is strongly $\mathcal{N} \mathscr{P}$-complete.

Proof. Analogous to the proof of Proposition 16. We consider the separation of circuit inequalities over the complete graph $K_{n}$, letting $d_{\{i, j\}}^{* *}:=2\left(1-1 / n-1 / n^{2}\right)$ if $\{i, j\} \in E$ and $d_{\{i, j\}}^{* *}:=2$ otherwise, and note that $d^{*}$ satisfies the triangle inequalities and that, again, there is a violated inequality if and only if $E$ contains a Hamiltonian circuit.

Moreover, we suspect that also for clique and bipartite inequalities separation is not easier when the projected version is considered.

Conjecture 2. The separation problem for projected clique inequalities is strongly $\mathcal{N} \mathscr{P}$-complete.

Conjecture 3. The separation problem for projected bipartite inequalities is strongly $\mathcal{N} \mathscr{P}$-complete.

Again, for the complexity of separation of projected tree inequalities, we do not dare to conjecture.

\subsection{On the Strength of Projected Inequalities}

As already mentioned, the projected version of a facet-inducing inequality is not even necessarily face inducing, i.e., with maximal right-hand side in " $\geq$ " form, as the example discussed next will show. Given that our method is widely based on the use of projected inequalities, this is an important issue to be addressed because, even if the separation of such inequalities is generally doable only for the original right-hand side, the right-hand side should be changed into maximal when they are added to the current LP.

Example 1 (Continued). The projected star inequality $2 d_{\{1,2\}}+d_{\{2,3\}}+2 d_{\{1,4\}}+d_{\{4,5\}}+2 d_{\{1,6\}}+d_{\{6,7\}} \geq$ 12 is not face inducing, because the minimum of the left-hand side over all feasible arrangements is in fact 13. With this value as the right-hand side, the inequality together with the trivial lower bounds would yield a value of 8 for the LP relaxation (6), equal to LA(G).

In this section, we discuss this issue for projected star inequalities, which are (by far) the most relevant inequalities in our method. For these inequalities, although computation of a maximal right-hand side appears to be nontrivial in general, we will derive a relatively simple sufficient condition for the original right-hand side to be maximal. Our computational experiments show that this condition is satisfied basically for all projected star inequalities separated, which implies that the original projected inequalities are fine in practice.

Consider a projected star inequality associated with $i \in V, S \subseteq N(i)$, and paths $P_{i j} \in \mathscr{P}_{i j}$ for $j \in S$ :

$$
\sum_{j \in S} \sum_{e \in P_{i j}} d_{e} \geq\left\lfloor(|S|+1)^{2} / 4\right\rfloor \text {. }
$$

First of all, we derive a suitable subclass of these inequalities that dominates the whole class. For each path $P_{i j}$, let $\vec{P}_{i j}$ denote the directed path obtained by orienting its edges from $i$ to $j$, as illustrated in Figure $1(b)$. We denote an edge $\{h, k\}$ oriented from $h$ to $k$ by $(h, k)$.

Proposition 18. The subclass of projected star inequalities (17) such that

(i) for each $j \in S$, if $P_{i j}$ contains an intermediate vertex $h \notin\{i, j\}$, we have that $h \in S$; and

(ii) $\bigcup_{j \in S} \vec{P}_{i j}$ induces a directed tree $T$ in $G$ (rooted at i); dominates the whole class of projected star inequalities.

PRoof. Consider a projected star inequality not satisfying (i), i.e., such that there exists a vertex $h$ visited by $P_{i j}$ for which $h \notin S$. This inequality is dominated as it can be obtained by summing together: (a) the inequality obtained by replacing $S$ by $S \backslash\{j\} \cup\{h\}$ and $P_{i j}$ by its subpath from $i$ to $h$, and (b) the (slack) lower bounds $d_{e} \geq 0$ for the edges in the subpath of $P_{i j}$ from $h$ to $j$. By iterating the procedure one gets a dominating inequality satisfying (i).

Now consider a projected star inequality satisfying (i) but not (ii), i.e., such that there exists a vertex $h \in S$ that is visited by a subpath $P_{i h}^{\prime} \subseteq P_{i j}$ for $j \neq h$ and $P_{i h}^{\prime} \neq P_{i h}$. This inequality is dominated as it can be obtained by summing together and dividing by two: (a) the inequality obtained by replacing $P_{i h}^{\prime}$ by $P_{i h}$ in the path from $i$ to $j$, and (b) the inequality obtained by replacing $P_{i h}$ by $P_{i h}^{\prime}$ in the path from $i$ to $h$. By iterating the procedure on the new inequalities until such a vertex $h$ does not exist, one obtains a collection of inequalities satisfying (i) and (ii) that dominate the original inequality. (In particular, note that, for all the inequalities in the collection, there is no edge $\{h, k\}$ that is oriented from $h$ to $k$ in one path and from $k$ to $h$ in another one.)

Observe that the proof of Proposition 18 yields a polynomial-time algorithm to construct, from a projected star inequality violated by the current LP solution, a violated inequality satisfying (i) and (ii). We now state the above-mentioned sufficient condition under which such an inequality is face inducing (which, as already mentioned, turns out to be almost always satisfied in practice). 
Proposition 19. Consider a projected star inequality satisfying (i) and (ii) in Proposition 18 , let $\{1, \ldots, s\}$ be the neighbors of $i$ in $T$ and, for $j=1, \ldots, s, T^{j}$ be the subtree of $T$ rooted at $j$. If there exists a partition of $\{1, \ldots, s\}$ into $S^{1}$ and $S^{2}$ such that $\left|\sum_{j \in S^{1}}\right| T^{j}\left|-\sum_{j \in S^{2}}\right| T^{j} \| \leq 1$, then the inequality is face inducing.

Proof. We let $w_{e}:=\left|\left\{j \in S: \vec{P}_{i j} \ni e\right\}\right|$ for each oriented edge $e \in T$ be the number of paths containing $e$. Moreover, for each vertex $h$ in $T$, we let $\delta_{T}^{+}(h)$ denote the set of edges leaving $h$ in T. Note that, if (ii) is satisfied, condition (i) is equivalent to stating that, for each oriented edge $(h, k) \in T$,

$$
w_{(h, k)}=1+\sum_{e \in \delta_{T}^{+}(k)} w_{e}
$$

(in words, out of all paths visiting $k$, one ends in $k$ and the other ones continue).

Note that the left-hand side of the inequality has the form $\sum_{e \in T} w_{e} d_{e}$; i.e., it can be seen as the objective function of the weighted variant of MinLA over a tree. We show that there exists a layout of the vertices in $T$ in which the value of this objective function is $\left\lfloor(|T|+1)^{2} / 4\right\rfloor$, which implies that the right-hand side is as large as possible. In fact, we show that this is the cost of any layout such that (a) the vertices in $\bigcup_{j \in S^{1}} T^{j}$ (respectively, $\bigcup_{j \in S^{2}} T^{j}$ ) appear to the left (respectively, right) of $i$ and (b) for $j \in S^{1}$ (respectively, $j \in S^{2}$ ), for each oriented edge $(h, k) \in T^{j}, h$ is to the right (respectively, left) of $k$.

Indeed, consider a layout satisfying (a) and (b). By keeping this layout and the set of vertices unchanged, we transform $T$ into a sequence of different trees for which the layout cost is the same, ending with a star having $i$ as a center and all edge weights equal to one, for which the cost of the layout is indeed the required value because, by (a), $\sum_{j \in S^{1}}\left|T^{j}\right|$ vertices are to the left of $i$ and $\sum_{j \in S^{2}}\left|T^{j}\right|$ vertices are to the right of $i$ (i.e., the same number of vertices, modulo one, are to the left and the right of the star center, meaning that the star layout is optimal).

If $w_{e}=1$ for $e \in T, T$ is already a star and there is nothing to be done. Otherwise, consider an oriented edge $(h, k) \in T$ such that $w_{(h, k)}>1$ (possibly with $h=i)$, along with another oriented edge $(k, l) \in T$, noting that such an edge exists by (18) (possibly with $\left.w_{(k, l)}=1\right)$. Recalling $(\mathrm{b})$, we focus on the case in which $h, k$, and $l$ appear in this order in the layout from left to right (i.e., $\{h, k, l\} \subseteq \bigcup_{j \in S^{2}} T^{j} \cup\{i\}$ ), the opposite case being identical. We remove from $T$ edge $(k, l)$, add to $T$ edge $(h, l)$ with $w_{(h, l)}:=w_{(k, l)}$, and redefine $w_{(h, k)}:=w_{(h, k)}-w_{(k, l)}$. It is easy to check that the new weights still satisfy (18) and that the cost of the layout is the same for the new tree (and weights). By iterating the procedure, we end up with a tree $T$ such that $w_{e}=1$ for $e \in T$.

\section{Computational Experiments}

Our algorithm was implemented in $C$ and run on a PC with processor Intel Core 2 Duo $3.33 \mathrm{GHz}$ and 2 GB of RAM under Microsoft Windows XP Professional Version 2002 SP2, using CPLEX 11.2 as the LP solver. As the results in this section show, our approach may be quite time consuming, so we imposed a time limit of one day (86,400 seconds) of CPU time for each instance.

\subsection{Separation Heuristics}

Triangle, path, (projected) star, and double star inequalities are separated efficiently in our code by the methods illustrated in $\S 4$. In this section, we illustrate the heuristic procedures used to separate the remaining inequalities for the complete graph $K_{n}$. According to Proposition 17, these procedures can be used also for their projected versions. The general impression is that it is fairly easy to find violated inequalities whatever the heuristic used.

For clique inequalities (8), we use a simple greedy heuristic that defines the clique $S$ by starting with the vertex $i$ such that $\sum_{j \in V \backslash\{i\}} d_{\{i, j\}}^{*}$ is minimum and then, at each iteration, adding the vertex $j$ such that a suitable weighted combination of $\sum_{i \in S} d_{\{i, j\}}^{*}$ and $\sum_{i \in V \backslash(S \cup\{j\})} d_{\{i, j\}}^{*}$ is minimum. For each set $S$ considered in the various iterations (and for its complement $V \backslash S$ ), we test violation of the corresponding clique inequality.

For bipartite inequalities (10), note that, once the set of $p$ vertices in one side of the bipartition is fixed, the separation is easy by using the same observations as in the separation of (double) star inequalities. Accordingly, we first enumerate all sets for $p=2$ (and $q$ even) and check the associated bipartite inequalities. Then, letting $S$ be the initially empty set of vertices on one side of the bipartition, we perform a sequence of iterations adding to $S$ the vertex such that $\sum_{i \in V \backslash(S \cup\{j\})} d_{\{i, j\}}^{*}$ is minimized, and if $p=|S|>2$, we check the associated bipartite inequalities.

We use two simple heuristic procedures for the separation of tree inequalities (14). In the first one, for each vertex $i \in V$, we consider each partial (shortest path) tree $T$ in one of the iterations of Dijkstra's algorithm with source $i$ and edge weights $d^{*}$, and check the tree inequality for $T$ (the right-hand side $\mathrm{LA}(T)$ is computed by our implementation of the algorithm in Shiloach 1979). The second procedure is analogous, considering each partial (shortest spanning) tree $T$ in one of the iterations of Prim's algorithm with source $i$ and edge weights $d^{*}$.

Finally, for circuit inequalities (9), we find a maximum-weight collection of vertex-disjoint circuits in $K_{n}$ (some of the vertices may not be contained in any circuit) by letting the weight of each edge $e$ be equal to $2-d_{e}^{*}$ (recall the proof of Proposition 16). 
Such a collection can be found efficiently by matching techniques. Then, we check the inequality associated with each circuit in the collection.

\subsection{Implementation Details}

We report the results for the LP relaxations in §2. In our cutting plane approach, we initialized these relaxations with the $n$ star inequalities (7) having, for $i \in V$, vertex $i$ as center and $S=N(i)$. When the dense LP relaxation (2) is solved, the clique inequality (8) with $S=V$ (given that $V$ itself is a clique of $K_{n}$ ) is also added.

We solve all the LPs in the cutting plane process by dual simplex. After the solution of each LP relaxation, we separate inequalities in the following order.

For the dense LP relaxation (2), the first inequalities that are separated (by complete enumeration) are the triangle inequalities (3). For the sparse LP relaxation (1) and the projected LP relaxation (6), we may or may not separate the path inequalities (5), because, according to the discussion in $\$ 2$ these inequalities do not improve the final lower bound if the time limit is not reached, but their explicit addition may speed up the solution process. We will indicate in the results if (5) were separated.

After having separated (3) in the dense case and, possibly, (5) in the projected case, we separate, in this order:

- star inequalities, by the exact algorithm in Proposition 13;

- clique inequalities, by the heuristic in $\$ 5.1$;

- circuit inequalities, by the heuristic in $\$ 5.1$;

- tree inequalities, by the two heuristics in \$5.1;

- double star inequalities, by the exact algorithm in Proposition 14; and

- bipartite inequalities, by the heuristic in \$5.1.

As the results below will show, the star inequalities, whose separation is very easy, are by far the most important ones in our approach. Moreover, for the projected LP relaxation, we often separate several projected star inequalities (17) associated with the same star inequality (7) of $K_{n}$. This is because the shortest path (according to distances $d^{*}$ ) from the center $i$ of the star to the vertices in $S$ changes from iteration to iteration. To limit this phenomenon, if there were violated projected star inequalities with center $i \in V$ for a given number of consecutive cutting plane iterations, before adding a further projected star inequality (17) associated with $i$ and $S \subseteq N(i)$, we consider all edges $e=\{i, j\}$ for $j \in S$, and (a) if $d_{e}$ is not yet a variable of the current LP (this is possible only if $\{i, j\} \notin E$ ), we add it; and (b) if not present, we add the path inequality (5) associated with edge $\{i, j\}$ and with the current shortest path $P_{i j}$ from $i$ to $j$. (Given that we may add path inequalities associated with edges not in $E$, this is important also in case we separate explicitly (5) for the edges in E.)

\subsection{Benchmark Instances}

We first considered the well-known instances from the Petit test set (Petit 2003b), available at http://www. lsi.upc.edu/ jpetit/MinLA/Experiments/. On the largest instances of this test set, the time limit was exceeded even when trying to solve the sparse LP relaxation (1) with $\mathscr{G}$ equal to the set of stars of $G$, which is essentially the simplest and fastest-to-compute lower bound discussed in this paper. Accordingly, we restricted our attention to the instances for which this lower bound could be computed. In fact, these are the instances with fewer than 3,000 edges (the next largest instance, random $A 1$, has almost 5,000 edges).

We also considered the bandwidth instances addressed in Caprara and Salazar-González (2005), available at http://www.informs.org/Journal/IJOC/ Online-Supplements/Volume-17-2005/Caprara-and-SalazarGonzalez-2005. Out of the associated connected graphs, we removed bcsstk02, as it is complete. Moreover, because all the results are identical for the six $e 05 r *$ instances and the two $r d b *$ instances (although the graphs are not, but they may be isomorphic), we give the results only for $e 05 r 0000$ and and $r d b 200$.

\subsection{Comparison of the LP Relaxations in \$2}

In Table 3 we compare the lower bounds found by the LP relaxations illustrated in $\S 2$, using star inequalities only. For the projected LP relaxation (6) we report the results with and without the explicit separation of the path inequalities (5).

Table 3 shows that the sparse LP relaxation (1) can be solved within a relatively short time but the lower bounds produced are fairly poor. The situation does not change substantially if, in addition to star inequalities, other inequalities are added to this relaxation. In other words, considering inequalities associated with subgraphs of the complete graph (rather than the specific graph considered) appears to be essential to finding good lower bounds.

Moreover, the table shows that the dense LP relaxation (2) and (3), which would produce the same lower bounds as (6) if solved to optimality, is much (a few orders of magnitude) slower to be solved, exceeding the time limit in all but two instances. For the cases in which (2) and (3) reaches the time limit, with the exception of instance $g d 96 b$, the lower bound it finds is much worse than the one found by (6).

Although it often reaches the time limit, the lower bounds produced by (6) are very good for instances $c * y$, for which the previous relative gap between bestknown heuristic and lower bound values was roughly $80 \%$, and fairly good for instances $g d *$, for which the previous relative gap was roughly $50 \%-75 \%$, with the exception of $g d 96 a$, for which it was roughly $95 \%$. The lower bound for bintree10 is not bad, whereas for 
Table 3 Lower Bounds and Running Times for the LP Relaxations in §2 with Star Inequalities Only

\begin{tabular}{|c|c|c|c|c|c|c|c|c|c|c|c|c|}
\hline \multirow[b]{2}{*}{ Name } & \multicolumn{3}{|c|}{$\mathrm{LP}(1)$} & \multicolumn{3}{|c|}{ LP (2) and (3) } & \multicolumn{3}{|c|}{$\mathrm{LP}(6)$} & \multicolumn{3}{|c|}{ LP (6) and (5) } \\
\hline & LB & Gap (\%) & Time & LB & Gap (\%) & Time & LB & Gap (\%) & Time & LB & Gap (\%) & Time \\
\hline$g d 95 c$ & 312 & 38.3 & 0.3 & 424 & 16.2 & $1,889.6$ & 424 & 16.2 & 0.3 & 424 & 16.2 & 0.3 \\
\hline gd96a & 6,708 & 93.0 & $2,237.8$ & 8,755 & 90.8 & limit & 70,674 & 25.8 & limit & 77,860 & 18.3 & limit \\
\hline gd96b & 1,199 & 15.3 & 1.5 & 1,199 & 15.3 & limit & 1,261 & 10.9 & 5.2 & 1,261 & 10.9 & 9.3 \\
\hline gd96c & 193 & 62.8 & 0.1 & 376 & 27.6 & $2,822.6$ & 376 & 27.6 & 1.2 & 376 & 27.6 & 0.5 \\
\hline gd96d & 920 & 61.5 & 4.6 & 912 & 61.9 & limit & 1,966 & 17.8 & 28.3 & 1,966 & 17.8 & 27.0 \\
\hline c1y & 25,431 & 59.1 & 293.7 & 27,093 & 56.5 & limit & 59,948 & 3.7 & $4,511.9$ & 59,948 & 3.7 & $10,259.8$ \\
\hline$c 2 y$ & 29,604 & 62.4 & 465.4 & 31,295 & 60.3 & limit & 76,229 & 3.2 & $5,617.3$ & 76,229 & 3.2 & $8,684.8$ \\
\hline c3y & 37,184 & 69.8 & 961.6 & 38,529 & 68.7 & limit & 113,801 & 7.6 & limit & 113,739 & 7.6 & limit \\
\hline$c 4 y$ & 28,137 & 75.5 & 869.2 & 29,528 & 74.3 & limit & 106,942 & 7.0 & limit & 106,627 & 7.2 & limit \\
\hline c5y & 29,773 & 69.3 & 687.7 & 31,237 & 67.7 & limit & 88,741 & 8.4 & limit & 86,755 & 10.4 & limit \\
\hline mesh $33 \times 33$ & 3,136 & 90.1 & 662.2 & 5,394 & 83.0 & limit & 19,691 & 37.8 & limit & 20,042 & 36.7 & limit \\
\hline bintree10 & 1,362 & 63.1 & 909.0 & 2,098 & 43.2 & limit & 2,847 & 23.0 & 47.1 & 2,847 & 23.0 & 45.9 \\
\hline
\end{tabular}

Note. Time limit of 86,400 seconds.

mesh $33 \times 33$ the solution may be far from convergence when the time limit is reached, although these two instances are of limited interest because their optimal value is known.

All in all, Table 3 clearly shows that (6) widely outperforms the other two. As for the explicit separation of (5), sometimes it pays off, notably for instance $g d 96 a$, and sometimes it does not. According to the present discussion, in the remainder of this section we will not consider LPs (1) and (2) and (3) any more, and we report the results found by (6) both without and with the explicit separation of (5).

\subsection{Results with the Addition of the Inequalities in $\$ 3$}

Table 4 presents the results of LP relaxation (6) when, besides star inequalities, all the other inequalities in $\S 3$ are separated by the heuristic procedures outlined above. For instance bintree10, the separation of tree inequalities leads to the optimal value (which is not surprising), and then the method keeps on finding violated inequalities until the time limit. For the four small $g d^{*}$ instances, the lower bound improvement is notable, although not impressive, but the running time increases by a couple of orders of magnitude. Note that our separation heuristics find plenty of violated inequalities, and our impression is that even with an exact separation the lower bounds would be similar. For instances $c 1 y$ and $c 2 y$, although about $90 \%$ of the time limit is available when the solution of the LP with star inequalities only is complete, the lower bound improvement from then to the time limit is negligible. Of course, there is no improvement for the instances for which the time limit was reached already by separating star inequalities only. In summary, the other inequalities - which are the most natural ones to consider in addition to stars and are facet inducing as well-give limited improvements for these instances and for the instances in which the
LP relaxation with stars only is solved within a tiny fraction of the time limit. This is not the case for the instances from Caprara and Salazar-González (2005), as mentioned in \$5.6.

\subsection{Results for the Instances in Caprara and Salazar-González (2005)}

To find a MinLA heuristic solution for the instances from Caprara and Salazar-González (2005), we applied a multistart local search procedure kindly provided to us by Gerd Reinelt (2009).

In Table 5, we report the results of LP relaxation of (6) for these instances, both restricting attention to star inequalities only and separating all the inequalities in §3. (Moreover, for these instances, models (1) and (2) and (3) give much poorer results, which are not reported. The only exception is instance bcsstk01, for which model (2) and (3) finds a lower bound value of 971 within minutes.)

These results show that the quality of our lower bounds is variable for these instances, with a relative gap ranging from $0 \%$ (we could solve to proven

Table 4 Lower Bounds and Running Times for LP Relaxation (6) with All Inequalities in §3

\begin{tabular}{|c|c|c|c|c|c|c|}
\hline \multirow[b]{2}{*}{ Name } & \multicolumn{3}{|c|}{$\operatorname{LP}(6)$} & \multicolumn{3}{|c|}{ LP (6) and (5) } \\
\hline & LB & Gap (\%) & Time & LB & Gap (\%) & Time \\
\hline$g d 95 c$ & 443 & 12.5 & 113.6 & 443 & 12.5 & 68.3 \\
\hline gd96a & 70,674 & 25.8 & limit & 77,860 & 18.3 & limit \\
\hline$g d 96 b$ & 1,281 & 9.5 & 493.5 & 1,281 & 9.5 & 889.8 \\
\hline gd96c & 402 & 22.5 & 218.1 & 402 & 22.5 & 390.3 \\
\hline gd96d & 2,021 & 15.5 & $1,669.0$ & 2,021 & 15.5 & $1,642.2$ \\
\hline c1y & 59,970 & 3 & limit & 59,971 & 3.6 & limit \\
\hline$c 2 y$ & 76,251 & 3.2 & limit & 76,253 & 3.2 & limit \\
\hline$c 3 y$ & 113,801 & 7.6 & limit & 113,739 & 7.6 & limit \\
\hline$c 4 y$ & 106,942 & 7.0 & limit & 106,627 & 7.2 & limit \\
\hline c5y & 88,741 & 8.4 & limit & 86,755 & 10.4 & limit \\
\hline mesh $33 \times 33$ & 19,691 & 37.8 & limit & 20,042 & 36.7 & limit \\
\hline bintree10 & 3,696 & 0.0 & limit & 3,696 & 0.0 & limit \\
\hline
\end{tabular}

Note. Time limit of 86,400 seconds. 
Table 5

Results for the Bandwidth Benchmark Graphs in Caprara and Salazar-González (2005)

\begin{tabular}{|c|c|c|c|c|c|c|c|c|c|c|c|c|c|c|}
\hline \multirow[b]{2}{*}{ Name } & \multirow[b]{2}{*}{$n$} & \multirow[b]{2}{*}{$m$} & \multicolumn{3}{|c|}{ LP (6) (star) } & \multicolumn{3}{|c|}{ LP (6) (all) } & \multicolumn{3}{|c|}{ LP (6) and (5) (star) } & \multicolumn{3}{|c|}{ LP (6) and (5) (all) } \\
\hline & & & LB & Gap (\%) & Time & LB & Gap (\%) & Time & LB & Gap $(\%)$ & Time & LB & Gap (\%) & Time \\
\hline bcspwr01 & 39 & 46 & 84 & 20.8 & 0.0 & 91 & 14.2 & 0.8 & 84 & 20.8 & 0.0 & 91 & 14.2 & 0.7 \\
\hline bcspwr02 & 49 & 59 & 130 & 19.3 & 0.1 & 144 & 10.6 & 2.0 & 130 & 19.3 & 0.0 & 144 & 10.6 & 1.8 \\
\hline bcspwr03 & 118 & 179 & 567 & 16.5 & 1.4 & 588 & 13.4 & 189.5 & 567 & 16.5 & 0.9 & 588 & 13.4 & 254.6 \\
\hline bcspwr04 & 274 & 669 & 3,623 & 23.0 & 789.2 & 3,696 & 21.4 & limit & 3,623 & 23.0 & 105.9 & 3,700 & 21.4 & limit \\
\hline bcsstk01 & 48 & 176 & 778 & 31.3 & 2.7 & 971 & 14.2 & $23,232.8$ & 778 & 31.3 & 1.7 & 972 & 14.1 & $38,481.1$ \\
\hline bcsstk02 & 66 & 2,145 & 35,937 & 25.0 & limit & 35,937 & 25.0 & limit & 35,937 & 25.0 & $6,908.2$ & 35,937 & 25.0 & limit \\
\hline bcsstk04 & 132 & 1,758 & 25,762 & 13.6 & $5,674.6$ & 27,518 & 7.7 & limit & 25,762 & 13.6 & $2,434.9$ & 27,569 & 7.5 & limit \\
\hline bcsstk05 & 153 & 1,135 & 9,614 & 13.1 & $1,710.6$ & 9,653 & 12.7 & limit & 9,614 & 13.1 & $1,742.1$ & 9,653 & 12.7 & limit \\
\hline can-24 & 24 & 68 & 179 & 14.8 & 0.1 & 203 & 3.3 & 2.8 & 179 & 14.8 & 0.1 & 203 & 3.3 & 3.4 \\
\hline can-61 & 61 & 248 & 1,083 & 4.7 & 3.7 & 1,119 & 1.6 & $1,221.4$ & 1,083 & 4.7 & & 1,119 & 1.6 & 538.0 \\
\hline can-62 & 62 & 78 & 171 & 19.3 & 0.1 & 187 & 11.8 & 4.2 & 171 & 19.3 & 1 & 187 & 11.8 & 5.1 \\
\hline can-73 & 73 & 152 & 797 & 27.5 & 6.8 & 971 & 11.7 & $2,016.8$ & 797 & 27.5 & 2.6 & 971 & 11.7 & $4,855.5$ \\
\hline can-96 & 96 & 336 & 1,609 & 40.5 & 154.2 & 2,105 & 22.1 & $27,786.0$ & 1,609 & 5 & 39.9 & 2,105 & 22.1 & $8,280.6$ \\
\hline can-144 & 144 & 576 & 1,752 & 45.7 & 142.2 & 2,304 & 28.5 & $19,608.4$ & 1,752 & 7 & 2.4 & 2,304 & 28.5 & $1,710.6$ \\
\hline can-161 & 161 & 608 & 4,568 & 31.8 & $1,788.9$ & 5,657 & 15.5 & limit & 4,568 & 3 & 628.9 & 5,569 & 16.8 & limit \\
\hline can-187 & 187 & 652 & 3,053 & 41.2 & $17,521.6$ & 3,827 & 26.2 & limit & 3,051 & 4 & limit & 3,051 & 41.2 & limit \\
\hline can-229 & 229 & 774 & 6,155 & 36.6 & $11,094.8$ & 7,461 & 23.1 & limit & 6,155 & 3 & $6,707.4$ & 7,461 & 23.1 & limit \\
\hline can-256 & 256 & 1,330 & 18,211 & 20.9 & $33,942.8$ & 20,627 & 10.4 & limit & 18,162 & 21.1 & limit & 18,162 & 21.1 & limit \\
\hline can-268 & 268 & 1,407 & 17,377 & 19.6 & limit & 17,377 & 19.6 & limit & 17,304 & 19.9 & limit & 17,304 & 19.9 & limit \\
\hline can-292 & 292 & 1,124 & 14,144 & 28.2 & $18,653.5$ & 14,981 & 24.0 & limit & 14,144 & 28.2 & $4,354.2$ & 15,012 & 23.8 & limit \\
\hline$d w t-59$ & 59 & 104 & 222 & 23.2 & 0.2 & 258 & 10.7 & 55.4 & 222 & 23.2 & 0.1 & 258 & 10.7 & 88.2 \\
\hline$d w t-66$ & 66 & 127 & 192 & 0.0 & 0.0 & 192 & 0.0 & 1.7 & 192 & 0.0 & 0.0 & 192 & 0.0 & 1.7 \\
\hline$d w t-72$ & 72 & 75 & 143 & 14.4 & 0.1 & 150 & 10.2 & 6.7 & 143 & 14.4 & 0.1 & 149 & 10.8 & 7.0 \\
\hline$d w t-87$ & 87 & 227 & 879 & 5.7 & 4.5 & 897 & 3.8 & $20,761.1$ & 879 & 5.7 & 3.8 & 897 & 3.8 & $18,430.7$ \\
\hline$d w t-162$ & 162 & 510 & 1,832 & 24.6 & $4,667.1$ & 2,032 & 16.4 & limit & 1,832 & 24.6 & $3,901.4$ & 2,029 & 16.5 & limit \\
\hline$d w t-193$ & 193 & 1,650 & 21,021 & 15.0 & $24,126.8$ & 23,073 & 6 & limit & 21,021 & 15 & 8,6 & 23,154 & 6.4 & limit \\
\hline$d w t-209$ & 209 & 767 & 5,731 & 10.3 & $2,001.5$ & 5 & 7. & limit & 5,731 & 10.3 & 51 & 5,905 & 7.5 & limit \\
\hline$d w t-221$ & 221 & 704 & 3,571 & 5.5 & $2,721.9$ & 3,603 & 4.7 & limit & 3,571 & 5.5 & 634.6 & 3,603 & 4.7 & limit \\
\hline$d w t-245$ & 245 & 608 & 3,403 & 11.8 & 382.1 & 3,422 & 11.3 & limit & 3,403 & 11.8 & 128.2 & 3,422 & 11.3 & limit \\
\hline bwm200 & 200 & 298 & 484 & 2.4 & 403.4 & 495 & 0.2 & $2,409.1$ & 484 & 2.4 & 234.4 & 495 & 0.2 & $33,409.2$ \\
\hline e05r0000 & 236 & 2,847 & 41,814 & 29.2 & limit & 41,814 & 29.2 & limit & 43,377 & 26.5 & limit & 43,377 & 26.5 & limit \\
\hline fidap001 & 216 & 2079 & 32,419 & 15.0 & limit & 32,419 & 15.0 & limit & 32,325 & 15.2 & limit & 32,325 & 15.2 & limit \\
\hline fidap005 & 27 & 126 & 402 & 2.9 & 0.2 & 412 & 0.5 & 5.4 & 402 & 2.9 & 0.2 & 412 & 0.5 & 4.2 \\
\hline fidapm05 & 42 & 239 & 975 & 2.8 & 2.3 & 998 & 0.5 & $7,492.9$ & 975 & 2.8 & 0.9 & 998 & 0.5 & 805.2 \\
\hline Ishp-265 & 265 & 744 & 5,137 & 15.6 & $13,761.3$ & 5,497 & 9.7 & limit & 5,137 & 15.6 & $22,256.4$ & 5,602 & 8.0 & limit \\
\hline lund-a & 147 & 1,151 & 9,489 & 16.2 & $6,679.3$ & 10,760 & 5.0 & limit & 9,489 & 16.2 & $2,530.1$ & 10,772 & 4.9 & limit \\
\hline lund- $b$ & 147 & 1,147 & 9,465 & 15.4 & $5,441.1$ & 10,692 & 4.5 & limit & 9,465 & 15.4 & $3,156.9$ & 10,712 & 4.3 & limit \\
\hline nos4 & 100 & 247 & 943 & 8.5 & 9.9 & 976 & 5.3 & $59,118.0$ & 943 & 8.5 & 5.7 & 976 & 5.3 & $60,497.5$ \\
\hline pde225 & 225 & 420 & 2,145 & 29.4 & 725.5 & 2,539 & 16.5 & $70,519.0$ & 2,145 & 29.4 & 287.4 & 2,538 & 16.5 & limit \\
\hline$r d b 200$ & 200 & 460 & 2,581 & 31.5 & $1,144.3$ & 3,052 & 19.0 & limit & 2,581 & 31.5 & 267.6 & 3,066 & 18.6 & $73,463.6$ \\
\hline saylr1 & 238 & 445 & 2,313 & 25.6 & $1,208.7$ & 2,673 & 14.0 & limit & 2,313 & 25.6 & 257.7 & 2,676 & 13.9 & limit \\
\hline steam1 & 240 & 1,761 & 21,017 & 26.3 & $57,429.7$ & 21,559 & 24.4 & limit & 21,017 & 26.3 & limit & 21,017 & 26.3 & limit \\
\hline steam3 & 80 & 424 & 1,360 & 4.0 & 30.8 & 1,382 & 2.4 & limit & 1,360 & 4.0 & 8.3 & 1,406 & 0.7 & limit \\
\hline tub100 & 100 & 148 & 236 & 4.1 & 1.9 & 245 & 0.4 & 74.3 & 236 & 4.1 & 0.2 & 245 & 0.4 & 71.5 \\
\hline
\end{tabular}

Note. Time limit of 86,400 seconds.

optimality instance $d w t-66)$ to about $35 \%$. Moreover, the quality of the bounds increases significantly with the addition of other inequalities besides stars, a notable difference with respect to the classical MinLA instances discussed so far. In any case, for about a third of the instances, the gap is less than $10 \%$, representing a first significant step toward proving that the solutions found by the common heuristics are near optimal.

\section{Conclusions}

The MinLA instances in the literature are a wellestablished benchmark for the problem. In this paper, we show for the first time that the best-known heuristic solutions in the literature are indeed near optimal for most of these instances. We hope that our contribution will stimulate further research in this direction. This result is obtained by combining two LP relaxations that were already tested previously with limited success, namely, the sparse LP relaxation (1) and the dense LP relaxation (2), into a unique projected LP relaxation (6) that combines the advantages of both.

LP relaxation (6) has the following two main limits. First of all, although we have put a lot of effort into speeding up the computation, for many instances even only solving the relaxation with star inequalities 
is very time consuming and essentially does not lead to any significant result for instances with more than 5,000 edges within one day of computation on a PC. Second, even with the addition of the integrality requirement on the variables, the model would not be a valid formulation of MinLA, and testing the MinLA feasibility of a given integer solution would be nontrivial (in fact, we conjecture that this is an $\mathcal{N} \mathscr{P}$-complete recognition problem).

To overcome the second limit above, in Caprara et al. (2009) a different formulation of the problem is proposed, with a much larger number of variables. The solution of the associated LP relaxation uses many of the ideas presented in this paper for the solution of LP (6). This approach could solve to proven optimality the two smallest instances in the MinLA benchmark, namely, gd95c and gd96c, and find better lower bounds than ours for $g d 96 b$ and $g d 96 d$. On the other hand, recalling the first limit above, this formulation seems to be absolutely too large for the other MinLA benchmark instances, as well as for any graph with more than a few hundred edges.

\section{Acknowledgments}

The second author was supported by the Engineering and Physical Sciences Research Council under Grant EP/D072662/1. The third author was supported by "Ministerio de Ciencia e Innovación" under Grant MTM2009-14039-C06-01. The authors are grateful to André Amaral for illuminating discussions and to Gerd Reinelt for providing them with the source code of a multistart local search procedure for MinLA implemented at the University of Heidelberg.

\section{References}

Amaral, A. R. S., A. N. Letchford. 2006. A polyhedral approach to the single row facility layout problem. Working paper, Lancaster University, Lancaster, UK.

Ambühl, C., M. Mastrolilli, O. Svensson. 2007. Inapproximability results for sparsest cut, optimal linear arrangement, and precedence constrained scheduling. Proc. 48th Annual IEEE Sympos. Foundations Comput. Sci., IEEE Computer Society Press, Los Alamitos, CA, 329-337.

Bornstein, C. F., S. Vempala. 2004. Flow metrics. Theoret. Comput. Sci. 321(1) 13-24.

Caprara, A., J. J. Salazar-González. 2005. Laying out sparse graphs with provably minimum bandwidth. INFORMS J. Comput. 17(3) 356-373.

Caprara, A., M. Jung, M. Oswald, G. Reinelt, E. Traversi. 2009. A betweenness approach for solving the linear arrangement problem. Working paper, DEIS, Università di Bologna, Bologna, Italy.

Charikar, M., M. T. Hajiaghayi, H. Karloff, S. Rao. 2010. $l_{2}^{2}$ spreading metrics for vertex ordering problems. Algorithmica 56(4) 577-604.

Chung, F. R. K. 1988. Labellings of graphs. L. Beineke, R. Wilson, eds. Selected Topics in Graph Theory 3. Academic Press, San Diego, 151-168.
Devanur, N. R., S. A. Khot, R. Saket, N. K. Vishnoi. 2006. On the hardness of minimum linear arrangement. Working paper, Georgia Institute of Technology, Atlanta.

Díaz, J., J. Petit, M. Serna. 2002. A survey of graph layout problems. ACM Comput. Surveys 34(3) 313-356.

Easton, T., S. B. Horton, R. G. Parker. 1996. A solvable case of the optimal linear arrangement problem on Halin graphs. Congressus Numerantium 119 3-17.

Even, G., J. S. Naor, S. Rao, B. Schieber. 2000. Divide-and-conquer approximation algorithms via spreading metrics. J. ACM 47(4) 585-616.

Feige, U., J. R. Lee. 2007. An improved approximation ratio for the minimum linear arrangement problem. Inform. Processing Lett. 101(1) 26-29.

Frederickson, G. N., S. E. Hambrush. 1988. Planar linear arrangements of outerplanar graphs. IEEE Trans. Circuits Systems 35(3) 323-332.

Garey, M. R., D. S. Johnson. 1979. Computers and Intractability: An Introduction to the Theory of $\mathcal{N} \mathscr{P}$-Completeness. Freeman, New York.

Garey, M. R., D. S. Johnson, L. J. Stockmeyer. 1976. Some simplified $\mathcal{N} \mathscr{P}$-complete graph problems. Theoret. Comput. Sci. 1(3) 237-267.

Grötschel, M., L. Lovász, A. J. Schrijver. 1988. Geometric Algorithms and Combinatorial Optimization. John Wiley \& Sons, New York.

Harper, L. H. 1964. Optimal assignments of numbers to vertices. SIAM J. Appl. Math. 12(1) 131-135.

Juvan, M., B. Mohar. 1992. Optimal linear labelings and eigenvalues of graphs. Discrete Appl. Math. 36(2) 153-168.

Koren, Y., D. Harel. 2002. A multi-scale algorithm for the linear arrangement problem. L. Kučera, ed. Proc. 28th Internat. Workshop Graph-Theoretic Concepts in Comput. Sci. Lecture Notes in Computer Science, Vol. 2573. Springer, Berlin, 293-306.

Liu, W., A. Vannelli. 1995. Generating lower bounds for the linear arrangement problem. Discrete Appl. Math. 59(2) 137-151.

Mitchison, G., R. Durbin. 1986. Optimal numberings of an $n \times n$ array. SIAM J. Discrete Math. 7(4) 571-582.

Nemhauser, G. L., L. A. Wolsey. 1988. Integer and Combinatorial Optimization. Wiley, Chichester, UK.

Muradyan, D. O., T. E. Piliposjan. 1980. Minimal numberings of vertices of a rectangular lattice. Akademii Nauk Armianskoi SSR 1(70) 21-27 (in Russian).

Petit, J. 2001. Layout problems. Ph.D. thesis, Department of Languages and Information Systems, Universitat Politècnica de Catalunya, Barcelona, Spain.

Petit, J. 2003a. Combining spectral sequencing and parallel simulated annealing for the MinLA problem. Parallel Processing Lett. 13(1) 77-91.

Petit, J. 2003b. Experiments on the linear arrangement problem. J. Experiment. Algorithmics 8 Article 2.3.

Rao, S., A. W. Richa. 2005. New approximation techniques for some linear ordering problems. SIAM J. Comput. 34(2) 388-404.

Reinelt, G. 2009. Personal communication.

Rodriguez-Tello, E., J.-K. Hao, J. Torres-Jimenez. 2008. An effective two-stage simulated annealing algorithm for the minimum linear arrangement problem. Comput. Oper. Res. 35(10) 3331-3346.

Safro, I., D. Ron, A. Brandt. 2006. Graph minimum linear arrangement by multilevel weighted edge contractions. J. Algorithms 60(1) 24-41.

Shiloach, Y. 1979. A minimum linear arrangement algorithm for undirected trees. SIAM J. Comput. 8(1) 15-32. 